\title{
Long-term survival in thymic carcinoma with postoperative pleural dissemination
}

\author{
Toru Kimura $^{1 *} \mathbb{0}$, Masahiko Higashiyama², Keiichiro Honma ${ }^{3}$, Harumi Nakamura ${ }^{4}$, Tomohiro Maniwa ${ }^{1}$ and \\ Jiro Okami ${ }^{1}$
}

\begin{abstract}
Background: We report a patient with thymic squamous cell carcinoma who underwent multiple rounds of surgical resection and definitive radiotherapy for both primary tumor and postoperative recurrence. However, the patient remains well and healthy 18 years after initial diagnosis. Since long-term survival after postoperative recurrence of thymic carcinoma is extremely rare, we also present her immunohistochemical staining results, which suggested indolent disease.
\end{abstract}

Case presentation: A 42-year-old woman with thymic squamous cell carcinoma underwent en bloc resection of the tumor and thymus gland. Pleural dissemination was noted in the right thoracic cavity 3, 10, and 16 years postoperatively. Where possible, the nodules were resected surgically: during the postoperative 3rd and 16th years. Definitive radiotherapy was administered for all nodules that could not be excised during the postoperative 3rd and 10th years. Disease-free survival is 25 months.

Conclusions: Local control of pleural dissemination may be beneficial in the treatment of postoperative recurrence of thymic carcinoma in limited cases of indolent disease.

Keywords: Thymic carcinoma, Postoperative recurrence, Disseminated pleural nodules, Long-term survival

\section{Background}

Thymic carcinoma is a rare and highly aggressive mediastinal neoplasm. Unlike in thymoma, the benefits of surgery and radiotherapy (RT) for pleural dissemination of thymic carcinoma remain unknown. Herein, we report an unusual case of long-term survival following multiple rounds of surgical resection and definitive RT for disseminated pleural nodules in a patient with thymic carcinoma.

\section{Case presentation}

A 42-year-old woman presented with a mediastinal tumor and anterior chest pain. Computed tomography $(\mathrm{CT})$ revealed a right anterior mediastinal tumor

\footnotetext{
*Correspondence: ruotora99@hotmail.com

1 Department of General Thoracic Surgery, Osaka International Cancer

Institute, 1,3,4: 3-1-69, Otemae, Chuo-ku, Osaka 541-8567, Japan

Full list of author information is available at the end of the article
}

measuring $7.0 \times 4.2 \times 3.2 \mathrm{~cm}$ (Fig. 1A). CT-guided coreneedle biopsy was performed. Since the tumor had been diagnosed as thymoma and estimated to be resectable at initial surgery, she underwent en bloc resection of the tumor and entire thymus gland through a full median sternotomy. Pathological examination revealed that the tumor was moderately differentiated thymic squamous cell carcinoma (SCC), which showed atypical cells varying in size and shape and proliferating in a sheet-like pattern (Fig. 1B). Immunohistochemical staining for $P A X 8$, CD5, $c-K I T$, TdT, and CD1a also demonstrated features that are characteristic to thymic carcinoma (Fig. 1C-G). The tumor was graded as pT2 N0 M0, p-Stage II according to the Union for International Cancer Control TNM eighth edition classification. During the third postoperative year, a follow-up CT demonstrated multiple nodules in the right thoracic cavity, which were characteristics of pleural dissemination. We resected all visible nodules on the visceral pleura except for a nodule with firm 


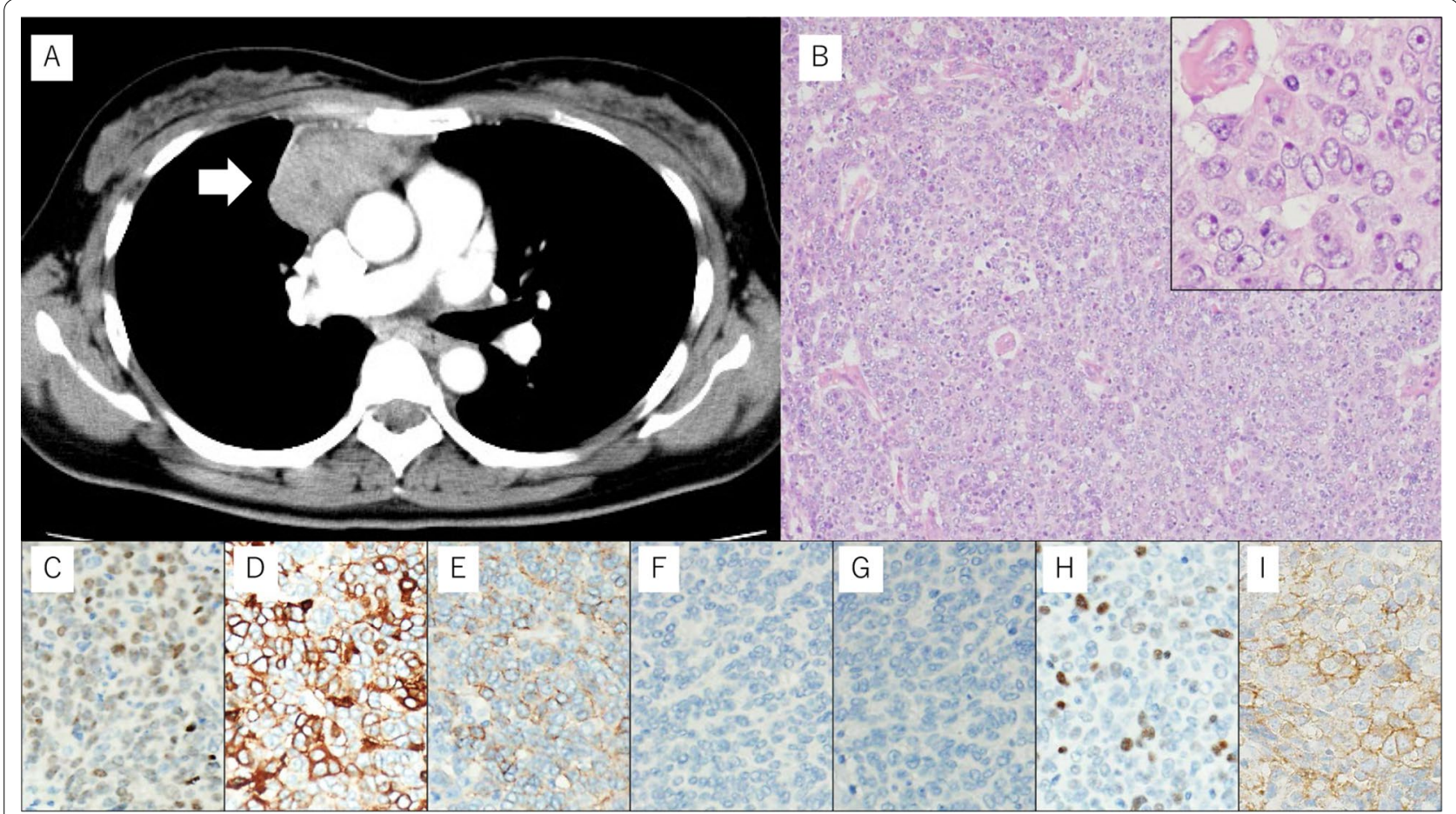

Fig. 1 A Chest computed tomography imaging of the primary tumor (arrow). B Pathological examination of the primary tumor reveals thymic squamous cell carcinoma. Hematoxylin and eosin staining; original magnification $\times 50$ and $\times 200$ (inset). C-I On immunohistochemical staining, the primary tumor is positive for PAX8 (C), CD5 (D), and c-KIT (E), while intratumoral lymphocytes are negative for TdT (F) and CD1a (G). The primary tumor demonstrates low expression of p53 (H) and positive staining for programmed death-ligand 1 (I). Original magnification $\times 200$

adhesions located on the anterior chest wall (Fig. 2A). We administered four courses of systemic chemotherapy with carboplatin and etoposide and delivered definitive $\mathrm{RT}$ at a dose of 60 Gy directly to the nodule. The patient developed rheumatic arthritis (RA) over the course of this treatment. During the tenth postoperative year, a follow-up CT revealed two nodules in the right thoracic cavity (Fig. 2B, C). We selected RT over surgical resection due to previous intraoperative findings in this patient. $\mathrm{RT}$ at a dose of $60 \mathrm{~Gy}$ was delivered to each of the nodules. During the 16th postoperative year, a follow-up CT revealed another pleural nodule (Fig. 2D), which was resected through a horizontal incision made just above the lesion. Histopathological examination of the resected pleural nodule confirmed establishing a similar diagnosis of thymic squamous cell carcinoma. Immunohistochemical staining for Ki-67 revealed that proliferation of tumor cells gradually increased (Fig. 3A-C). Immunohistochemical staining for p53 and programmed deathligand 1 (PD-L1 using 22C3 anti-PD-L1 antibody) in the primary tumor (Fig. 1H, I) and this nodule demonstrated that both were positive for wild-type TP53 and PD-L1. Twenty-five months following the last surgery, the patient remained in good health. No new lesions were noted. Her $\mathrm{RA}$ is well controlled with medication.

\section{Discussion}

Studies of thymic carcinoma have consistently demonstrated a significant survival advantage in surgically treated patients (1). Multidisciplinary treatment with surgical resection, chemotherapy, and RT improves prognosis even in cases of advanced disease or incomplete resection $[1,2]$. Comparatively, prognosis remains poor in patients with postoperative recurrence of thymic carcinoma [3]. In a report by Hamaji et al. [3] in which they evaluated nine patients with recurrent thymic carcinoma, none survived more than 5 years.

Unlike thymomas, thymic carcinomas are infrequently associated with autoimmune disorders, such as myasthenia gravis and RA [4]. The clinical course of metachronous disseminated pleural nodules coupled with a long-term survival rate in our patient is also more peculiar to thymoma rather than to thymic carcinoma [3, 4]. However, immunohistochemical evaluation, including expression patterns of PAX8, CD5, $c-K I T, \mathrm{Td} \mathrm{T}$, and CD1a that are characteristic to thymic carcinoma [5], confirmed the diagnosis in our patient. None of the recurrent pleural nodules were shown to have originated from a thymoma or any other organ. Next-generation sequencing has demonstrated that TP53 is the most frequently mutated gene in thymic 

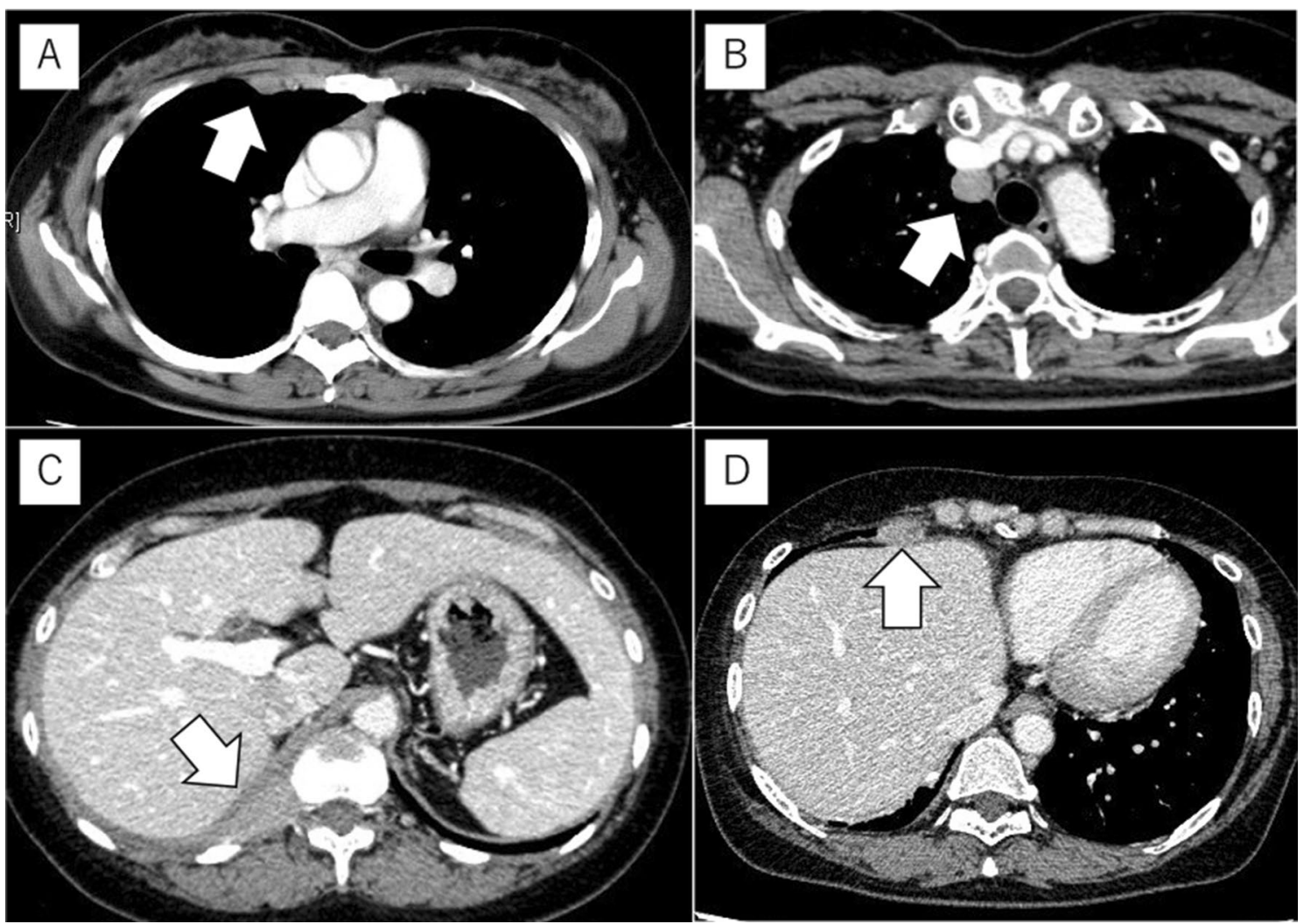

Fig. 2 Chest computed tomography (CT) imaging of the disseminated pleural nodules seen in the postoperative recurrence of thymic carcinoma (arrows). A CT taken during the 3rd postoperative year. A nodule is noted on the anterior chest wall. B, C CT taken during the 10th postoperative year. A nodule is observed right behind the right brachiocephalic vein (B) and at the crus of the diaphragm (C). D CT taken during the 16th postoperative year. A nodule is shown between the anterior chest wall and the diaphragm

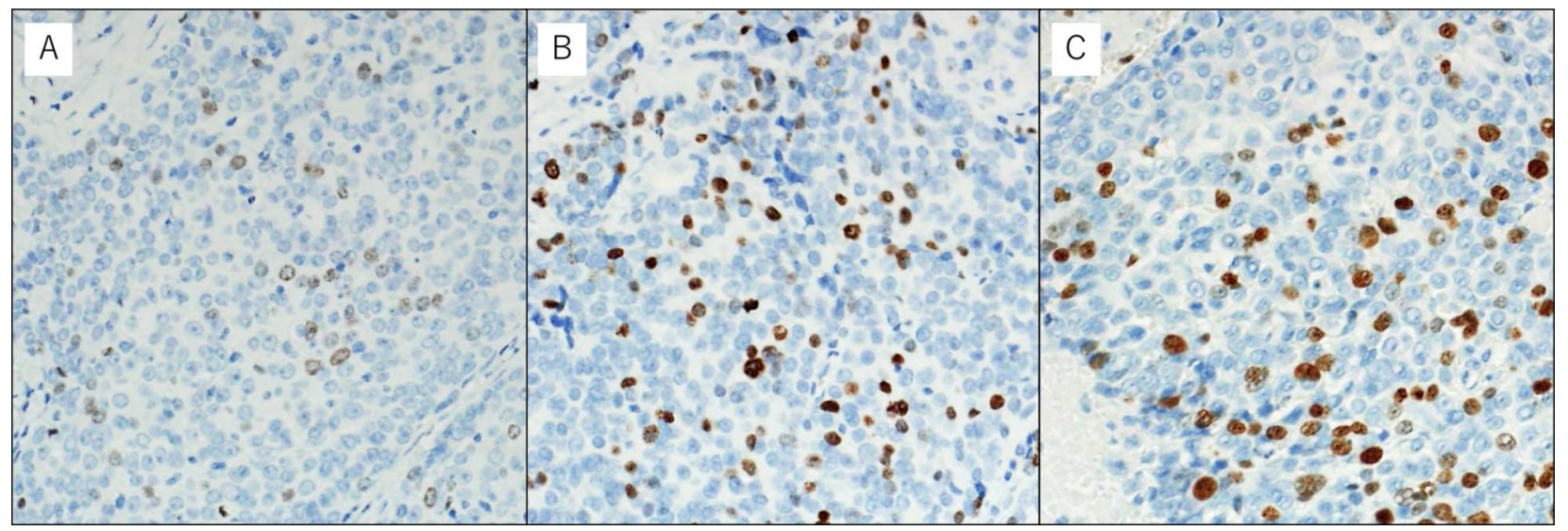

Fig. 3 Immunohistochemical staining of the primary tumor and disseminated nodules for Ki-67. The fraction of Ki-67-positive tumor cells in the primary tumor $(\mathbf{A})$, disseminated nodule at the third postoperative year (B), and that at the 16 th postoperative year $(\mathbf{C})$ were $10 \%, 15 \%$, and $25 \%$, respectively. Original magnification $\times 200$

cancer. Alterations in p53 have also been shown to be associated with a worse prognosis [6]. The present case demonstrated low-level expression of p53 in less than
$50 \%$ of tumor cells [6], which may be compatible with a favorable prognosis in our patient. While PD-L1 positivity in thymic carcinomas has been reportedly shown 
to be $41 \%-100 \%$ [7], there is no consensus on whether high PD-L1 expression in thymic carcinoma is associated with better [8] or worse [9] outcomes. T cells expressing the programmed death-1 (PD-1) co-receptor have also been correlated with the pathogenesis of RA [10]. However, correlation between PD-L1 expression on thymic epithelial tumors and RA as a comorbidity is still not well understood. Additional research on PD-L1 expression and its biological significance in thymic carcinoma is needed due to the rarity of this disease.

Surgical resection has been shown to result in favorable long-term outcomes in patients with disseminated pleural nodules from both thymoma [11] and postoperative recurrence of thymic carcinoma [3, 12]. Prognosis of thymic carcinoma is further improved with neoadjuvant and adjuvant RT, as well as concurrent or sequential RT and chemotherapy [13]. Successful RT as part of the multidisciplinary treatment of thymic carcinoma suggests that thymic carcinoma may have cellular sensitivity to RT. However, only a limited number of case reports have documented this success to date [14, 15]. Our findings suggest that surgical resection and definitive RT of pleural nodules may contribute to better outcomes in selected cases of thymic carcinoma.

\section{Conclusion}

We encountered a rare case of long-term survival in a patient who underwent multiple rounds of surgical resection and RT for primary thymic carcinoma and its subsequent recurrences. Local control of pleural dissemination may be beneficial for the treatment of postoperative recurrent thymic carcinoma in limited cases with indolent disease.

\section{Abbreviations}

CT: Computed tomography; PD-L1: Programmed death-ligand 1; RA: Rheumatic arthritis; RT: Radiotherapy.

\section{Acknowledgements}

None declared.

\section{Authors' contributions}

TK: conceptualization, resources, writing-original draft; $\mathrm{MH}$ : resources, writing - review and editing, supervision; $\mathrm{KH}$ : resources, supervision; HN: resources, supervision; TM: writing — review and editing, supervision; JO: writing - review and editing, supervision. All authors read and approved the final manuscript.

\section{Funding}

This study did not receive any funding from private, public, or not-for-profit agencies.

\section{Availability of data and materials}

Data sharing is not applicable to this article as no datasets were generated or analyzed during the current study.

\section{Declarations}

Consent for publication

Not applicable.

\section{Competing interests}

The authors declare that they have no competing interests.

\section{Author details}

${ }^{1}$ Department of General Thoracic Surgery, Osaka International Cancer Institute, 1,3,4: 3-1-69, Otemae, Chuo-ku, Osaka 541-8567, Japan. ${ }^{2}$ Department of General Thoracic Surgery, Higashiosaka City Medical Center, 2: 3-4-5, Nishiiwata, Higashiosaka, Osaka 578-8588, Japan. ${ }^{3}$ Department of Pathology, Osaka International Cancer Institute, 1,3,4: 3-1-69, Otemae, Chuo-ku, Osaka 541-8567, Japan. ${ }^{4}$ Laboratory of Genomic Pathology, Osaka International Cancer Institute, 1,3,4: 3-1-69, Otemae, Chuo-ku, Osaka 541-8567, Japan.

Received: 9 May 2021 Accepted: 19 July 2021

Published online: 30 July 2021

\section{References}

1. Ruffini E, Detterbeck F, van Raemdonck D, Rocco G, Thomas P, Weder W, et al. Thymic carcinoma: a cohort study of patients from the European society of thoracic surgeons database. J Thorac Oncol. 2014;9:541-8.

2. Okuma Y, Horio H, Hosomi Y, Watanabe K, Maeda Y, Okamura T, et al. The potency of curative-intent treatment for advanced thymic carcinoma. Lung Cancer. 2014:84:175-81.

3. Hamaji M, Allen MS, Cassivi SD, Nichols FC 3rd, Wigle DA, Deschamps C, et al. The role of surgical management in recurrent thymic tumors. Ann Thorac Surg. 2012;94:247-54.

4. Kelly RJ, Petrini I, Rajan A, Wang Y, Giaccone G. Thymic malignancies: from clinical management to targeted therapies. J Clin Oncol. 2011;29:4820-7.

5. Asirvatham JR, Esposito MJ, Bhuiya TA. Role of PAX-8, CD5, and CD117 in distinguishing thymic carcinoma from poorly differentiated lung carcinoma. Appl Immunohistochem Mol Morphol. 2014;22:372-6.

6. Moreira AL, Won HH, Mcmillan R, Huang J, Riely GJ, Ladanyi M, et al. Massively parallel sequencing identifies recurrent mutations in TP53 in thymic carcinoma associated with poor prognosis. J Thorac Oncol. 2015:10:373-80.

7. Owen D, Chu B, Lehman AM, Annamalai L, Yearley JH, Shilo K, et al. Expression patterns, prognostic value, and intratumoral heterogeneity of PD-L1 and PD-1 in thymoma and thymic carcinoma. J Thorac Oncol. 2018;13:1204-12.

8. Yokoyama S, Miyoshi H, Nakashima K, Shimono J, Hashigutchi T, Mitsuoka $M$, et al. Prognostic value of programmed death ligand 1 and programmed death 1 expression in thymic carcinoma. Clin Cancer Res. 2016;22:4727-34.

9. Padda SK, Riess JW, Schwartz EJ, Tian L, Kohrt HE, Neal JW, et al. Diffuse high intensity PD-L1 staining in thymic epithelial tumors. J Thorac Oncol. 2015:10:500-8

10. Rao DA, Gurish MF, Marshall JL, Slowikowski K, Fonseka CY, Liu Y, et al. Pathologically expanded peripheral Thelper cell subset drives B cells in rheumatoid arthritis. Nature. 2017;542:110-4.

11. Kimura K, Kanzaki R, Kimura T, Kanou T, Ose N, Funaki S, et al. Long-term outcomes after surgical resection for pleural dissemination of thymoma. Ann Surg Oncol. 2019;26:2073-80.

12. Bott MJ, Wang H, Travis W, Riely GJ, Bains M, Downey R, et al. Management and outcomes of relapse after treatment for thymoma and thymic carcinoma. Ann Thorac Surg. 2011:92:1984-92.

13. Ahmad U, Yao X, Detterbeck F, Huang J, Antonicelli A, Filosso PL, et al. Thymic carcinoma outcomes and prognosis: results of an international analysis. J Thorac Cardiovasc Surg. 2015;149(95-100):101.e1-2.

14. Ogawa K, Toita T, Uno T, Fuwa N, Kakinohana Y, Kamata M, et al. Treatment and prognosis of thymic carcinoma a retrospective: analysis of 40 cases. Cancer. 2002;94:3115-9.

15. Yamamoto Y, Kodama K, Maniwa T, Kishima H. Successful treatment of advanced thymic carcinoma with lymph node and pleural metastases: a case report. Mol Clin Oncol. 2016:5:550-2.

\section{Publisher's Note}

Springer Nature remains neutral with regard to jurisdictional claims in published maps and institutional affiliations. 